\title{
Werden die Raucher zu einer schützenswerten Minderheit?
}

Max Geiser

* Möglicherweise empfanden die beiden Politiker das Rauchen als Hilfe beim Durchstehen ihrer belastenden Arbeit.

Korrespondenz:

Prof. Dr. med. Max Geiser

Gossetstrasse 49

CH-3084 Wabern
In der medizinischen Literatur finden sich viele Untersuchungen, die einen Zusammenhang («association») zwischen dem Lungenkarzinom, einer Reihe von anderen Karzinomen und Krankheiten [1] und dem Tabakkonsum nahelegen. Für die einen Autoren besteht die einzige vernünftige Erklärung darin, dass Rauchen ein kausaler Faktor bei der Entstehung dieser Krankheiten ist und sich die möglichst frühzeitige Entwöhnung lohnt [1,2]. Andere Autoren lassen die Erklärung für den Zusammenhang offen [3].

Von Zeit zu Zeit erscheinen in der Presse Artikel, die den Tabakkonsum für die verschiedensten Krankheiten verantwortlich machen. Von Tausenden und Millionen «Zigarettentoten» ist die Rede. Nach der erfolgten Publikation im «British Medical Journal» [2] hat ein Redaktor der «NZZ am Sonntag» vom 25. Juli 2004 einen staatlichen Nichtraucherschutz gefordert [4] und ein Schriftsteller hat in der gleichen Ausgabe eine «rauchfreie Zukunft» prophezeit [5].

Die bei britischen Ärzten während 50 Jahren durchgeführte Studie über deren Rauchverhalten mit Hilfe von Fragebogen zeigt nicht mehr und nicht weniger als die statistisch gesicherte Tatsache, dass Tabakkonsum in Abhängigkeit vom Ausmass und von der Dauer ein Faktor unter anderem bei der Entstehung von Lungenkarzinomen, von Krankheiten der Atemwege und kardio- und zerebrovaskulären Krankheiten ist und dass zigarettenrauchende britische Ärzte durchschnittlich zehn Jahre früher sterben als nichtrauchende Ärzte. Ferner führt ein Konsumstopp zur Verlängerung der Lebenserwartung, die um so grösser ist, je früher der Rauchstopp realisiert wird. Der Laienleser weiss natürlich nicht, dass die Aussagekraft von Fragebogenstudien durch zahlreiche Mängel wie fehlende Kontrollier- und Reproduzierbarkeit, unzulängliche Repräsentativität, ungenaue Antworten und Unsicherheit bei der Todesursachendiagnose ohne histologischen Beweis [6] geschmälert ist. Das Bundesamt für Gesundheit BAG führt seine Kampagne für eine «rauchfreie Gesellschaft» seit mehr als zehn Jahren durch und kämpft gleichzeitig ebensolang für die Straffreiheit des Rauschgiftkonsums. Die Mannschaft im BAG kennt natürlich die wissenschaftliche Unsicherheit von Studien mit Hilfe von Fragebo- gen, die vor allem für die Marktforschung, aber kaum für die Ursachenforschung taugen. Trotz aller festgestellter Schädlichkeit des Tabakmissbrauchs kann niemand übersehen, dass die Eliminierung des Tabaks bestenfalls die Mortalität in der Sterblichkeitsstatistik von einer Kategorie zur anderen schiebt, weil wir alle, Raucher und Nichtraucher, sterben und die Langlebigkeit der Nichtraucher diese nicht unfehlbar glücklicher macht als früher sterbende Raucher.

Antitabakkampagnen sind aus mehreren Gründen fragwürdig:

1. Wenn die Raucher statistisch durchschnittlich zehn Jahre jünger sterben als die Nichtraucher, entlasten sie wahrscheinlich das Gesundheitswesen, weil sie weniger lange oder gar nicht unter den im hohen Alter auftretenden, noch nicht heilbaren Krankheiten, wie z.B. an Demenz, Parkinson, Behinderungen im Bewegungsapparat und Blindheit, leiden. Untersuchungen über die Belastung des Gesundheitswesens durch die dem Tabakkonsum angelasteten Krankheiten der früher sterbenden Raucher und durch die Alterskrankheiten der länger lebenden Nichtraucher fehlen.

2. Raucher speisen die AHV und erhalten Arbeitsplätze.

3. Im Gegensatz zu den Alkohol- und Drogensüchtigen verlieren Raucher ihren Verstand und ihre Leistungsfähigkeit nicht. Raucher, besonders Pfeifenraucher, sind oft sympathische, gemütvolle Mitbürger, die anständigerweise niemanden beim Essen und bei der Arbeit mit Rauch belästigen.

4. Da die statistische Wahrscheinlichkeit des früheren Sterbens bei weitem nicht alle und nicht einmal alle schweren Raucher trifft, starb Winston Churchill Gott sei Dank trotz seines jahrelangen Tabakkonsums nicht vorzeitig. Und der Zigarettenkettenraucher Helmut Schmidt lebt 86jährig immer noch, war ein herausragender Bundeskanzler und beeindruckt heute in Interviews durch seine weisen Antworten auf dumme Fragen.*

Die zurzeit laufende Kampagne für eine tabakfreie Gesellschaft und staatlichen Schutz für Nichtraucher hat totalitären Charakter mit ge- 
schichtlichen Vorläufern. Der türkische Sultan Murat IV. erklärte das Rauchen zum Kapitalverbrechen. Jakob I. fürchtete um die Ehre und Sicherheit des Königs und des Commonwealth. Adolf Hitler war überzeugt, dass die Tatsache, dass er Nichtraucher war, ihn widerstandsfähig mache und dies dem Heil des deutschen Volkes diene. Wäre er als Raucher vor der Machtübergabe gestorben, wäre der Welt viel erspart worden.

Obwohl Raucher die AHV speisen, Arbeitsplätze erhalten und durch ihr frühes Sterben Rentenversicherungen und möglicherweise das Gesundheitswesen finanziell entlasten, sind sie zurzeit in Gefahr, als gesellschaftsschädigende Minderheit verachtet $\mathrm{zu}$ werden, weil gesundheitsfanatische Beamte und Journalisten nicht imstande sind, die Unzulänglichkeiten von epidemiologischen Fragebogenstudien zu erkennen und die Fragwürdigkeit von Statistiken und deren Nichtanwendbarkeit auf das einzelne Individuum zu respektieren. Angesichts der durchschnittlichen Langlebigkeit müssten die Nichtraucher ethisch geradezu als Egoisten getadelt werden, weil sie Pensionskassen und Rentenversicherungen und das Gesundheitswesen länger belasten als die früher sterbenden Raucher, die ausserdem noch während vieler Jahre die AHV unterstützten. Die Nichtraucher zu schützen, ist sicher keine Kernaufgabe des Staates. Die Be- deutung des Risikofaktors Passivrauchen ist für die Verschlechterung der Lungenfunktion bei der chronisch obstruktiven Lungenkrankheit COPD weiterhin unklar [7]. Es ist eine Aufgabe der genetischen Forschung, herauszufinden, welche Individuen gefährdet sind, ein Lungenkarzinom oder eine COPD zu entwickeln, wenn sie rauchen. Der Lösung dieser Aufgabe ist die biomedizinische Wissenschaft näher denn je.

\section{Literatur}

1 Cornuz J, Humair JP, Zellweger JP. Tabakentwöhnung. Schweiz Med Forum 2004;4:764-70.

2 Doll R, Peto R, Boreham J, Sutherland I. Mortality in relation to smoking: 50 years observation on male British doctors. BMJ 2004;328:1519-28.

3 Doll R, Hill AB. The mortality of doctors in relation to their smoking habits; a preliminary report. Br Med J 1954;26:1451-5.

4 Hollenstein P. Nichtraucherschutz ist eine Kernaufgabe des Staates. NZZ am Sonntag, 25.7.2004. S.13.

5 Bilton P. Die Zukunft ist rauchfrei. NZZ am Sonn$\operatorname{tag}$ 25.7.2004. S.14.

6 Geiser M. Todesursachenstatistik und Gesundheitspolitik. Schweiz Ärztezeitung 1990;71:1887-90.

7 Dietel M, Suttorp N, Zeitz M (Hrsg.). Harrisons Innere Medizin. 16. Auflage. Berlin: ABW Wissenschaftsverlagsgesellschaft mbH; 2005. Band 2, S. 1659. 